\title{
Characteristics and Outcome of Patients After Allogeneic Hematopoietic Stem Cell Transplantation Treated With Extracorporeal Membrane Oxygenation for Acute Respiratory Distress Syndrome*
}

\author{
Philipp Wohlfarth, $\mathrm{MD}^{1}$; Gernot Beutel, $\mathrm{MD}^{2}$; Pia Lebiedz, $\mathrm{MD}^{3}$; Hans-Joachim Stemmler, PhD ${ }^{4}$; \\ Thomas Staudinger, $\mathrm{MD}^{1}$; Matthieu Schmidt, $\mathrm{PhD}^{5}$; Matthias Kochanek, $\mathrm{MD}^{6}$; Tobias Liebregts, $\mathrm{MD}^{7}$; \\ Fabio Silvio Taccone, $\mathrm{PhD}^{8}$; Elie Azoulay, $\mathrm{PhD}^{9}$; Alexandre Demoule, $\mathrm{PhD}^{10,11}$; Stefan Kluge, $\mathrm{MD}^{12}$; \\ Morten Svalebjørg, $\mathrm{MD}^{13}$; Catherina Lueck, $\mathrm{MD}^{2}$; Johanna Tischer, $\mathrm{MD}^{4}$; Alain Combes, $\mathrm{PhD}^{5}$; \\ Boris Böll, $\mathrm{MD}^{6}$; Werner Rabitsch, $\mathrm{MD}^{1}$; Peter Schellongowski, $\mathrm{MD}^{1}$ on behalf of Intensive Care in \\ Hematologic and Oncologic Patients (iCHOP) and the Caring for Critically Ill Immunocompromised \\ Patients Multinational Network (NINE-I)
}

\begin{abstract}
*See also p. 925.
${ }^{1}$ Department of Medicine I, Intensive Care Unit 13i2, Medical University of Vienna, Vienna, Austria.

${ }^{2}$ Department of Hematology, Hemostaseology, Oncology and Stem Cell Transplantation, Hannover Medical School, Hannover, Germany.

${ }^{3}$ Department of Cardiovascular Medicine, University Hospital Muenster, Muenster, Germany.

${ }^{4}$ Department of Medicine III, Intensive Care Unit I3, LMU Munich, Campus Großhadern, Munich, Germany.

${ }^{5}$ Medical Intensive Care Unit, Institute of Cardiometabolism and Nutrition, Hôpital de la Pitié-Salpêtrière, Assistance Publique-Hôpitaux de Paris, Université Pierre et Marie Curie, Paris, France.

${ }^{6}$ Department of Internal Medicine I, University of Cologne, Cologne, Germany.

${ }^{7}$ Department of Bone Marrow Transplantation, University Hospital Essen, West German Cancer Center, University of Duisburg-Essen, Essen, Germany.

${ }^{8}$ Department of Intensive Care, Hopital Erasme, Université Libre de Bruxelles, Brussels, Belgium.

${ }^{9}$ Medical Intensive Care Unit, Saint-Louis Teaching Hospital, Paris, France.

${ }^{10}$ Service de Pneumologie et Réanimation Médicale, Groupe Hospitalier Pitié Salpêtrière, Assistance Publique-Hôpitaux de Paris, Paris, France.

${ }^{11}$ Sorbonne Universités, UPMC Univ Paris 06, INSERM, UMRS1158 Neurophysiologie respiratoire expérimentale et clinique, Paris, France.

${ }^{12}$ Department of Intensive Care Medicine, University Medical Center Hamburg-Eppendorf, Hamburg, Germany.

${ }^{13}$ Department of Anaesthesiology, Oslo University Hospital, Oslo, Norway. Drs. Wohlfarth and Schellongowski contributed to the conception of the study, data collection, data analysis and interpretation, and drafted the article. Drs. Staudinger and Rabitsch contributed to the conception of the study, data interpretation, and revised the article critically for important intellectual content. Drs. Beutel, Lebiedz, Stemmler, Schmidt, Kochanek, Liebregts, Taccone, Azoulay, Demoule, Kluge, Svalebjørg, Lueck, Tischer, Copyright (c) 2017 by the Society of Critical Care Medicine and Wolters Kluwer Health, Inc. All Rights Reserved.
\end{abstract}

DOI: $10.1097 / C C M .0000000000002293$
Combes, and Böll contributed to data collection and interpretation, and revised the article critically for important intellectual content. All authors approved the final version of the article.

Supplemental digital content is available for this article. Direct URL citations appear in the printed text and are provided in the HTML and PDF versions of this article on the journal's website (http://journals.lww.com/ccmjournal).

Dr. Staudinger received funding from lecturing for Maquet and Xenios. He reports personal fees from Maquet and personal fees from Novalung, both outside the submitted work. Dr. Schmidt reports personal fees from Maquet, outside the submitted work. Dr. Kochanek received funding from Astellas Pharma, Pfizer Pharma, and Gilead Pharma. Dr. Azoulay received funding from lecturing for Astellas, Alexion, Cubist, and Gilead. His institution received funding from grants from Gilead, Astellas, Fisher \& Paykel, and Alexion. Dr. Demoule received funding from Maquet, and other support from MSD and Covidien (lectures). His institution received funding from Covidien and Philips. $\mathrm{He}$ reports grants and personal fees from Maquet, personal fees and nonfinancial support from Covidien, grants and nonfinancial support from Philips, nonfinancial support from Drager, personal fees from MSD, and grants from Air Liquide Santé, all outside the submitted work. Dr. Kluge received support for article research from the National Institutes of Health $(\mathrm{NIH})$, Wellcome Trust/COAF, Austrian Science Fund (FWF), Howard Hughes Medical Institute (HHMI), Bill \& Melinda Gates Foundation, World Bank, and Research Councils UK (RCUK). He received funding from NovaLung (lectures and as a member of advisory board) and Gambro/Baxter (lectures and as a member of advisory board). He reports personal fees from Novalung, outside the submitted work. Dr. Combes reports financial support by Maquet related to the EOLIA trial, outside the submitted work. Dr. Schellongowski received funding from Maquet, Novalung, and Astellas. He disclosed other support from Amgen, Astellas, Baxter, Fisher \& Paykel, Hill-Rom, Jazz Pharmaceuticals, MSD, and Pfizer. He reports personal fees from Maquet and personal fees from Novalung, both outside the submitted work. The remaining authors have disclosed that they do not have any potential conflicts of interest.

For information regarding this article, E-mail: peter.schellongowski@ meduniwien.ac.at

Objectives: The acute respiratory distress syndrome is a frequent condition following allogeneic hematopoietic stem cell transplantation. Extracorporeal membrane oxygenation may serve as 
rescue therapy in refractory acute respiratory distress syndrome but has not been assessed in allogeneic hematopoietic stem cell transplantation recipients.

Design: Multicenter, retrospective, observational study.

Setting: ICUs in 12 European tertiary care centers (Austria, Germany, France, and Belgium).

Patients: All allogeneic hematopoietic stem cell transplantation recipients treated with venovenous extracorporeal membrane oxygenation for acute respiratory distress syndrome between 2010 and 2015.

Interventions: None.

Measurements and Main Results: Thirty-seven patients, nine of whom underwent noninvasive ventilation at the time of extracorporeal membrane oxygenation initiation, were analyzed. ICU admission occurred at a median of 146 (interquartile range, 27-321) days after allogeneic hematopoietic stem cell transplantation. The main reason for acute respiratory distress syndrome was pneumonia in $81 \%$ of patients. All but one patient undergoing noninvasive ventilation at extracorporeal membrane oxygenation initiation had to be intubated thereafter. Overall, seven patients (19\%) survived to hospital discharge and were alive and in remission of their hematologic disease after a follow-up of 18 (range, 5-30) months. Only one of 24 patients (4\%) initiated on extracorporeal membrane oxygenation within 240 days after allogeneic hematopoietic stem cell transplantation survived compared to six of 13 $(46 \%)$ of those treated thereafter $(p<0.01)$. Fourteen patients (38\%) experienced bleeding events, of which six (16\%) were associated with fatal outcomes.

Conclusions: Discouraging survival rates in patients treated early after allogeneic hematopoietic stem cell transplantation do not support the use of extracorporeal membrane oxygenation for acute respiratory distress syndrome in this group. On the contrary, longterm allogeneic hematopoietic stem cell transplantation recipients otherwise eligible for full-code ICU management may be potential candidates for extracorporeal membrane oxygenation therapy in case of severe acute respiratory distress syndrome failing conventional measures. (Crit Care Med 2017; 45:e500-e507)

Key Words: acute respiratory distress syndrome; extracorporeal membrane oxygenation; respiratory insufficiency; stem cell transplantation

\footnotetext{
$\Lambda$ llogeneic hematopoietic stem cell transplantation (ASCT) is increasingly performed to treat various malignant and nonmalignant hematologic diseases. Reports from the United States (1) and Europe (2) mention the respective record numbers of 8,000 and 16,000 ASCTs carried out in 2014. The occurrence rate of the acute respiratory distress syndrome (ARDS) was recently reported to be as high as $16 \%$ within the first year following ASCT, and three out of four of these patients present with severe $\operatorname{ARDS}(3,4)$. In the light of these numbers, ASCT recipients with severe ARDS may be a quantitatively relevant target population to consider for treatment with extracorporeal membrane oxygenation (ECMO). Hence, specific recognition of these patients by critical care professionals seems mandatory.
}

The use of ECMO in ASCT recipients has so far only been reported as single case or in small series (5-10). Results from these reports together with the unfavorable outcome seen in conventional mechanical ventilation have driven discussions about the futility of ECMO in the setting of ASCT $(11,12)$. However, given the limited available data, there is a paucity of evidence to guide strict reasoning. With this study, we set out to report the characteristics and outcome of ASCT recipients treated with ECMO for ARDS in 12 European centers. The provided data are intended to strengthen clinical decision making about the use of ECMO under certain circumstances or in specific subgroups of patients following ASCT.

\section{MATERIALS AND METHODS}

We retrospectively studied the clinical courses of all adult ASCT recipients (18 yr old or older) treated with venovenous ECMO for ARDS in the ICUs of 12 European tertiary care centers (one respectively from Austria, Belgium, and Norway, three from France, and six from Germany) between 2010 and 2015. The study was approved by the ethics committee of the Medical University of Vienna and conducted in accordance with Good Clinical Practice guidelines and the amended Declaration of Helsinki. The term "ECMO" refers to venovenous ECMO throughout the article. Two of the patients were part of an already published case series (5).

Baseline data were recorded for the time immediately before initiation of ECMO therapy. ARDS was defined and graded according to the Berlin definition (4).The presence of circulatory, renal, or liver dysfunction at baseline was defined as a Sequential Organ Failure Assessment score (13) greater than or equal to 2 in the respective domain. The Simplified Acute Physiology Score II (SAPS II) $(14,15)$ at ICU admission was used to assess the severity of acute illness, the Charlson Comorbidity Index (CCI) (16) at admission to account for comorbidities, and the Lung Injury Score (17) to determine the extent of lung damage in invasively ventilated patients at baseline. Driving pressure $(\Delta \mathrm{P})$ in patients undergoing invasive mechanical ventilation (IMV) was calculated as described elsewhere (18).

Bleeding was defined as requirement of two or more units of packed RBCs due to an obvious bleeding event, and noted as major if a surgical or interventional procedure was required, as well as in cases of intracerebral hemorrhage or fatal outcome. Neutropenia was defined as an absolute neutrophil count less than 500 per microliter. In patients with more than one episode of ECMO, baseline variables associated with the first ECMO episode were analyzed, whereas all episodes were included in the calculation of the total duration of ECMO therapy.

Continuous data are presented as median and interquartile range $(25-75 \%)$, dichotomous data as number and percentage. Receiver operating characteristic analysis was used to identify the time from ASCT to initiation of ECMO to best discriminate between survivors and nonsurvivors and was then rounded to the nearest tenth not changing the properties of the discriminator. Data were compared between survivors and nonsurvivors using Fisher exact test for dichotomous variables and the Mann-Whitney $U$ test for continuous variables. 
Differences were considered statistically significant when $p$ value was less than 0.05 . Statistical tests were performed using the SPSS 22 software package (IBM, Armonk, NY).

\section{RESULTS}

Thirty-seven ASCT recipients with ARDS treated with a total of 39 ECMO episodes were included. The male-to-female ratio was 1.2:1, age $37(26-49)$ years, CCI $0(0-1)$, and SAPS II 56 (42-67). ICU admission occurred 139 (20-301) days after stem cell transplantation. Sixteen patients (43\%) were admitted to the ICU and had not been temporarily discharged after ASCT. Individual patient characteristics are depicted in the Supplementary Table 1 (Supplemental Digital Content 1, http://links. lww.com/CCM/C383).

\section{Characteristics of the Hematopoietic Stem Cell Transplantation}

ASCT-related characteristics are described in Table 1. Hematologic malignancy was the underlying disease in 34 patients

\section{TABLE 1. Allogeneic Hematopoietic Stem Cell Transplantation-Related Characteristics}

\section{Variable

Underlying condition

Acute leukemia

22 (59)

Lymphoma

5 (14)

Myelodysplastic syndrome

Other malignant condition

4 (11)

Nonmalignant disease

3 (8)

Conditioning therapy ${ }^{a}$

Myeloablative

Nonmyeloablative

7 (21)

Stem cell source

Peripheral blood

Bone marrow

Cord blood

Donor type

Unrelated donor

Related donor

14 (38)

Remission status at ICU admission

Complete remission

No remission, after engraftment

No remission, prior engraftment

5 (15)

GvHD at ICU admission

Acute GvHD

Chronic GvHD

8 (22)

Immunosuppressive therapy during ICU

Any immunosuppressive therapy

Corticosteroids

Calcineurin inhibitor

8 (22)

Mycophenolate mofetil

$5(14)$

Others
GvHD = graft versus host disease

aData are missing for three patients (two survivors and one nonsurvivor).

${ }^{b}$ Given for patients with malignant underlying conditions.

Data are presented as absolute numbers (\%).

$20(67)$

8 (27)

5 (17)

$$
\begin{gathered}
21(70) \\
5(17) \\
0 \\
2(7) \\
2(7)
\end{gathered}
$$

1 (14)

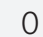

3 (43)

2 (29)

1 (14)

0.27

3 (60)

2 (40)

6 (86)

1 (14)

0

$22(79)$

5 (83)

2 (7)

0

4 (14)

1 (17)

5 (17)

0

0.56

6 (20)

2 (29)

0.63

28 (93)

3 (43)

0.006823
0 
(92\%), most frequently acute leukemia ( $n=22,59 \%)$. At ICU admission, signs of active graft versus host disease (GvHD) were documented in 13 patients (35\%) (acute, $n=5$; chronic, $n=8)$, and 31 patients ( $84 \%)$ received at least one immunosuppressive therapy at any time during their ICU stay. All but two patients were in complete remission of their underlying hematologic malignancy. Five patients had undergone ASCT without being in complete remission and were admitted prior to engraftment or first evaluation of remission.

\section{Characteristics of the Acute Respiratory Failure}

ARDS was related to pneumonia in 30 patients (81\%), abdominal sepsis and alveolar hemorrhage in two patients each, and transfusion-related acute lung injury, vasculitis, and bronchiolitis obliterans in one patient each. Microbiologic workup revealed possible pathogens in 25 patients $(68 \%)$, with Cytomegalovirus $(n=9,24 \%)$ and Pneumocystis jirovecii $(n=5$, $14 \%)$ in respiratory material having been the most frequent findings (Supplementary Table 1, Supplemental Digital Content 1, http://links.lww.com/CCM/C383). All patients undergoing IMV at ECMO initiation $(n=28,76 \%)$ fulfilled the criteria for severe ARDS ( $\mathrm{PaO}_{2} / \mathrm{FIO}_{2}$ ratio, 66 [49-80] mm Hg; positive end-expiratory pressure [PEEP], 12 [9-15] $\mathrm{cm} \mathrm{H}_{2} \mathrm{O}$; $\Delta \mathrm{P}, 20$ [17-24] mm Hg). Neuromuscular blocking agents, prone positioning, and nitric oxide therapy had been used as concomitant therapy for ARDS prior to ECMO in 14 (50\%), 10 (36\%), and seven $(25 \%)$ patients, respectively. ECMO was initiated in nine patients (24\%) with failing noninvasive mechanical ventilation (NIV; respiratory rate, 32 [30-35] per minute; $\mathrm{PaO}_{2} / \mathrm{FIO}_{2}$ ratio, 82 [57-98]; PEEP, 7 [5-10] $\mathrm{cm} \mathrm{H}_{2} \mathrm{O}$ ) (Table 2).

\section{ECMO Therapy and Complications}

ECMO was initiated 146 (27-321) days following ASCT, 4 (1-7) days after ICU admission, and 2 (0-6) days after intubation. All but one patient undergoing NIV at baseline were intubated 5 (2-9) days after start of ECMO. Lowest leukocyte counts observed were $0.9(0.2-2.7) \mathrm{G} / \mathrm{L}$ with periods of neutropenia recorded in 18 patients (49\%), and lowest platelet counts 8 (5-17) G/L with episodes of thrombocytopenia less than $50 \mathrm{G} / \mathrm{L}$ in $92 \%(n=34)$ of patients. During ECMO, 78\% $(n=29)$ and $51 \%(n=19)$ of patients required vasopressors and renal replacement therapy, respectively.

Bleeding events were documented in 14 patients $(38 \%)$; six $(16 \%)$ of these were regarded major. Latter comprised intracranial hemorrhage leading to discontinuation of active therapy and fatal multiple organ failure involving uncontrolled bleeding in three patients each (patients 4, 8, 17, 24, 27, and 29 [Supplementary Table 1, Supplemental Digital Content 1, http://links.lww.com/CCM/C383]). During ECMO, 14 patients were withheld from anticoagulation due to thrombocytopenia (median platelet count at initiation, $17 \mathrm{G} / \mathrm{L}$; range, 2-50 G/L), the remainder received heparin. Bleeding events occurred in four of 14 when compared with 10 of 23 in the heparin group $(p=0.49)$, one clotting event was noted in the former. We did not find significant differences of the platelet counts at ECMO baseline or during the course of ECMO treatment between patients with and without bleeding events (data not shown). Ischemic stroke was noted in three patients during ECMO with one progressing to intracerebral hemorrhage (included into the above stated patients). Thus, in all, five patients (14\%) experienced major cerebral complications during ECMO. No events of accidental decannulation were reported.

\section{Outcome and Survival Comparison}

Survival of the last ECMO episode was 9/37 (24\%). Two patients successfully weaned from ECMO died in the ICU. The ICU and hospital survival rate was 7/37 (19\%), and all survivors were alive and in remission of their disease 18 (range, 5-30) months after ICU discharge.

ECMO was initiated after a median time of more than 1 year following ASCT in hospital survivors (485 [270-976] vs 100 [24-226] d in nonsurvivors; $p=0.01$ ). A cutoff of 240 days from ASCT to ECMO was best discriminative regarding survival $(1 / 24[4 \%]$ vs $6 / 13$ [46\%]; $p<0.01)$. A significantly higher proportion of early ASCT recipients (i.e., started on ECMO within $240 \mathrm{~d}$ from ASCT) had two or more organ dysfunctions at baseline when compared with long-term ASCT recipients $(63 \%$ vs $15 \%$; $p=0.01)$. Regardless of the time span between ASCT and ECMO, less than half of the survivors received immunosuppressive therapies ( $43 \%$ vs 93\%; $p=0.01$ ), and none of the five patients with acute GvHD survived. Underlying conditions leading to ASCT varied between survivors and nonsurvivors $(p<0.01)$. Survival in NIV patients was $2 / 9(22 \%)$ when compared with $5 / 28(18 \%)$ in patients undergoing IMV at ECMO initiation $(p=1.0)$. A univariate comparison of characteristics between survivors and nonsurvivors are described in Tables 1 and 2, and comparisons between early and long-term ASCT recipients are depicted in Supplementary Table 2 (Supplemental Digital Content 1, http://links.lww.com/CCM/C383).

\section{DISCUSSION}

In this study, we analyzed the characteristics and outcomes of 37 ASCT recipients treated with ECMO for ARDS in 12 European centers. The ICU survival rate was 19\%, which is markedly lower than those reported in non-ASCT patients with ARDS undergoing ECMO $(19,20)$. Notably, there was a distinct and significant difference in the survival rates of patients treated early or late after ASCT (4\% vs 46\%). All survivors were discharged from the hospital, alive and in remission after long-term follow-up.

Several studies on heterogeneous ASCT populations undergoing IMV due to acute respiratory failure of all degrees have currently reported on survival rates of approximately $30 \%$ $(3,21,22)$. However, in the so far largest study on outcomes of more than 1,000 mainly hematologic patients with ARDS, the estimated 90-day mortality of those with "severe" ARDS exceeded $80 \%$, and ASCT status was an independent risk factor for even higher mortality (23). Despite these data, we found it difficult to match our findings with proper comparison groups, as ASCT patients with severe ARDS have so far not been characterized in detail. This is somewhat surprising, since 
according to a recent investigation, every sixth patient within 1 year after ASCT develops ARDS (3). ECMO in our study was initiated as a rescue therapy in patients with severe ARDS combined with multiple organ dysfunction, previously reported fatal $(21,24-27)$. Nevertheless, with respect to important prognostic variables, our cohort did not seem to differ from the mentioned non-ECMO ASCT cohorts, as we found rather low rates of nonremission status, acute GvHD, and invasive mold infections $(3,21,22)$. This reflects adherence to expert recommendations on ICU admission criteria for patients after ASCT

\section{TABLE 2. ICU and Extracorporeal Membrane Oxygenation-Related Characteristics and Outcome}

Variable

Characteristics at ICU admission

Age, yr

Sex, female

Charlson Comorbidity Index (16)

Simplified Acute Physiology Score II score

Days from allogeneic hematopoietic stem cell transplantation to ECMO

Characteristics at ECMO baseline

Invasive mechanical ventilation patients

$$
\begin{aligned}
& \text { Lung Injury Score } \\
& \text { PEEP, } \mathrm{cm} \mathrm{H}_{2} \mathrm{O} \\
& \text { Driving pressure }(\Delta \mathrm{P}), \mathrm{cm} \mathrm{H}_{2} \mathrm{O} \\
& \mathrm{PaO}_{2} / \mathrm{FIO}_{2} \text { ratio } \\
& \mathrm{pH} \\
& \mathrm{PaCO}_{2}, \mathrm{~mm} \mathrm{Hg}
\end{aligned}
$$

Noninvasive mechanical ventilation patients

Respiratory rate, breaths/min

PEEP, $\mathrm{cm} \mathrm{H}_{2} \mathrm{O}$

$\mathrm{PaO}_{2} / \mathrm{FIO}_{2}$ ratio

$\mathrm{pH}$

$\mathrm{PaCO}_{2}, \mathrm{~mm} \mathrm{Hg}$

All patients

$\begin{array}{lc}\text { Lactate, mM } & 1.7(1.2-3.7) \\ \text { Hemoglobin, g/dL } & 8.9(8.3-10.2) \\ \text { Leukocytes, G/L } & 3.5(1.0-8.3) \\ \text { Platelets, G/L } & 34(14-49)\end{array}$

Nonpulmonary organ dysfunctions at baseline $^{a}$

0

1

$$
\begin{aligned}
& 37(26-49) \\
& 17(46) \\
& 0(0-1) \\
& 56(42-67) \\
& 146(27-321) \\
& 28(76) \\
& 3.5(3.25-3.75) \\
& 12(9-15) \\
& 20(17-24) \\
& 66(49-80) \\
& 7.27(7.17-7.35) \\
& 61(52-72) \\
& 9(24)
\end{aligned}
$$

32 (30-35)

7 (5-10)

82 (57-98)

7.35 (7.28-7.45)

47 (34-62)

$$
\begin{aligned}
& 1.7(1.2-3.7) \\
& 8.9(8.3-10.2) \\
& 3.5(1.0-8.3) \\
& 34(14-49)
\end{aligned}
$$

1 (3)

\section{All Patients $(n=37)$}

Nonsurvivors $(n=30)$

Survivors $(n=7)$

p

$$
\begin{aligned}
& 36(28-49) \\
& 15(50) \\
& 0(0-1) \\
& 55(41-66) \\
& 100(24-226) \\
& 23(77) \\
& 3.5(3.25-3.75) \\
& 12(8-15) \\
& 21(17-24) \\
& 67(52-80) \\
& 7.27(7.13-7.35) \\
& 60(52-71) \\
& 7(23)
\end{aligned}
$$

$$
\begin{gathered}
38(26-58) \\
2(29)
\end{gathered}
$$$$
1(1-1)
$$$$
56 \text { (47-70) }
$$$$
485 \text { (270-976) }
$$

0.011

$$
5(71)
$$

1.0

$\begin{array}{cc}3.75(3.38-3.75) & 0.65 \\ 14(11-18) & 0.38 \\ 18(17-22) & 0.45 \\ 51(42-89) & 0.64 \\ 7.21(7.19-7.37) & 0.86 \\ 65(54-112) & 0.29 \\ 2(29) & 1.0\end{array}$




\section{TABLE 2. (Continued). ICU and Extracorporeal Membrane Oxygenation-Related Characteristics and Outcome}

Characteristics during ECMO

\begin{tabular}{|c|c|c|c|c|}
\hline Vasopressors & $29(78)$ & $24(80)$ & $5(71)$ & 0.63 \\
\hline Hemofiltration & $19(51)$ & $16(53)$ & $3(43)$ & 0.69 \\
\hline Bleeding event & $14(38)$ & $12(40)$ & $2(29)$ & 0.69 \\
\hline Neutropenia & $18(49)$ & $15(50)$ & $3(43)$ & 1.0 \\
\hline Lowest platelets, G/L & $8(5-17)$ & $8(5-14)$ & $8(2-54)$ & 0.69 \\
\hline Packed red cells $(0-5 / 5-10 />10)^{b}$ & $8(23) / 11(31) / 16(46)$ & $6(21) / 9(32) / 13(46)$ & $2(29) / 2(29) / 3(43)$ & 1.0 \\
\hline Platelet transfusions $(0-5 / 5-10 />10)^{b}$ & $11(31) / 9(26) / 15(41)$ & $7(25) / 9(32) / 12(43)$ & $4(57) / 0 / 3(43)$ & 0.15 \\
\hline \multicolumn{5}{|l|}{ Outcome } \\
\hline Duration of ECMO therapy, $d$ & $15(8-23)$ & $15(8-23)$ & $10(4-13)$ & 0.20 \\
\hline ICU length of stay, $d$ & $28(14-33)$ & $22(12-35)$ & $28(25-49)$ & 0.28 \\
\hline ICU and hospital survival & 7 (19) & & & \\
\hline
\end{tabular}

$\mathrm{ECMO}=$ extracorporeal membrane oxygenation; PEEP $=$ positive end-expiratory pressure.

aLiver, kidney, and cardiovascular dysfunction according to a Sequential Organ Failure Assessment score (13) $\geq 2$ in the respective category. No statistical comparisons were made for NIV patients due to small numbers.

${ }^{b}$ Data for 35 patients, transfusion data cover overall ICU stay.

Data are presented as medians (interquartile range) or absolute numbers (\%).

in our group (28) and, furthermore, suggests that our cohort may compare as a subgroup representative of ASCT recipients currently admitted to ICUs of dedicated centers (21).

As the major finding, our data indicate that a significant proportion of long-term ASCT recipients with refractory severe ARDS may be candidates for ECMO therapy, whereas prognosis of patients early after ASCT is very grim. Several aspects may explain this finding. First, immunocompromised state, a well-known risk factor in large ECMO cohorts $(29,30)$, gradually improves over time following ASCT and is accompanied by tapering of immunosuppressive medication (31). Second, acute GvHD predisposes to multiple organ dysfunction associated with detrimental outcome in critical illness $(21,24,25)$. In line with this, none of the patients with acute GvHD in our cohort survived. Third, idiopathic noninfectious pulmonary complications after ASCT, such as diffuse alveolar hemorrhage or idiopathic pneumonia syndrome, have been associated with excess mortality rates $(32,33)$, and may have been underreported in our cohort given the number of patients with microbiologically negative pneumonia around engraftment.

In contrast, the question can be raised when or whether previous ASCT may be regarded a less important detail rather than a major contributor to morbidity and mortality. Indeed, four survivors were admitted more than 1 year after ASCT, and two of these neither displayed signs of chronic GvHD nor received immunosuppressive therapies. The majority of long-term ASCT survivors presented with pulmonary infections and identified pathogens, both factors associated with favorable outcome in hematologic patients with respiratory failure $(23,34)$. Available data on ASCT patients do not give compelling evidence that the underlying malignancy itself (in contrast to the remission status) correlates with survival in case of critical illness $(3,21,25)$. Therefore, the difference in our series between survivors and nonsurvivors with respect to the underlying disease must be interpreted with extreme caution and in the light of small patient numbers.

Importantly, all but three patients developed pronounced thrombocytopenia during ECMO. Bleeding occurred frequently and was deemed major in $16 \%$, including three fatal cases of intracranial hemorrhage. Considering ischemic stroke in two additional patients raises the rate of critical cerebral complications to $14 \%$. Albeit bleeding remains a major issue, we recommend to withhold anticoagulation in patients with deep thrombocytopenia on heparinized circuits, as performed in several of our patients and recently presented in small series $(5,35)$.

Surprisingly, only half of the IMV patients received neuromuscular blockers and only a third underwent prone positioning. Hence, evidence-based adjunctive measures had not been fully exploited in all patients, which prompts speculation whether their use could have obviated ECMO in some cases. Although this is definitely a limitation to the interpretation of our findings, the apparent underuse of these measures in clinical practice likely also affects findings from other observational and even interventional ECMO trials $(36,37)$. As a contributing factor in our series, the respective landmark trials were just published during the study period $(38,39)$, and complete translation of their findings could, thus, not be expected.

In consequence to the low success rate and the high burden of complications, we feel that ECMO should be discouraged for ARDS during the peri- or early posttransplant period. In the remaining ASCT patients, we suggest to strictly reserve its 
use to cases of severe ARDS failing protective ventilation (40) together with supportive therapeutic measures, such as prone positioning (38) and neuromuscular blocking agents (39). In addition, we recommend 1) to very carefully select patients with preferably no additional organ dysfunctions and no signs of refractory acute GvHD, 2) to early inform patients and families about the limited prognosis, and 3) to discuss and respect their preferences with regards to end-of-life issues as a cornerstone of the decision process.

Conservative considerations also apply to the use of ECMO in patients with NIV failure for preventing endotracheal intubation. Based on encouraging findings from a small and uncontrolled cohort study (41), nine of our patients were placed on ECMO while undergoing failing NIV. However, all but one had to be intubated eventually. Thus, our preliminary data do not suggest feasibility of the "awake ECMO" concept in ASCT patients.

The formal limitations of our report are the ones typically associated with retrospective analyses and cover data completeness, quality, and interpretation. Most importantly, we can neither exclude unmeasured confounders nor present a control group or uniform ECMO inclusion criteria. However, all of our patients undergoing IMV would have fulfilled the strict inclusion criteria of the so far only positive interventional ECMO trial with respect to clinical outcome (37), and thus, may be considered ECMO candidates to the best available evidence. Furthermore, we did not provide extended data on or a uniform strategy for ECMO and ventilator settings, which, unfortunately, is a common limitation of most observational and interventional ECMO trials. Likewise, questions regarding risks other than bleeding, measures to avoid complications, and the role of an "awake ECMO" concept cannot be answered by our study. Even though we report the by far largest number of ASCT patients on ECMO, numbers are rather small, dictating cautious interpretation of statistical findings. Furthermore, the observational design does not allow for proofing causal relations between ECMO and outcome. Next steps to overcome the persisting lack of evidence in the management of ASCT patients with severe ARDS warrant better characterizations of outcome and prognostic factors, which may serve as a basis for well designed case-control studies or interventional protocols including ECMO in selected subgroups of patients.

\section{CONCLUSIONS}

Discouraging survival rates in patients treated early after ASCT do not support the use of ECMO for ARDS in this group. On the contrary, long-term ASCT recipients otherwise eligible for full-code ICU management may be potential candidates for ECMO therapy in case of severe ARDS failing conventional measures.

\section{ACKNOWLEDGMENTS}

Collaborators (alphabetically by country and institution) for both Intensive Care in Hematologic and Oncologic Patients (iCHOP) and the Caring for Critically Ill
Immunocompromised Patients Multinational Network (NINE-I) are listed below:

Austria: Medical University of Vienna (Andja Bojic [iCHOP, NINE-I], Alexander Hermann [iCHOP, NINE-I], Wolfgang Lamm [iCHOP, NINE-I], Werner Rabitsch [iCHOP, NINE-I], Oliver Robak [iCHOP, NINE-I], Peter Schellongowski [iCHOP, NINE-I], Wolfgang R. Sperr [iCHOP, NINE-I], Thomas Staudinger [iCHOP, NINE-I], Philipp Wohlfarth [iCHOP, NINE-I]); Belgium: Université Libre de Bruxelles (Anne-Pascale Meert [NINE-I], Fabio Silvio Taccone [NINE-I]); France: Hôpital de la Pitié-Salpêtrière (Alain Combes [NINE-I], Matthieu Schmidt [NINE-I]), Saint-Louis Teaching Hospital (Elie Azoulay [NINE-I], Virginie Lemiale [NINE-I]), Sorbonne Universités UPMC Univ Paris 06 (Alexandre Demoule [NINE-I]); Germany: University of Cologne (Michael von Bergwelt-Baildon [iCHOP, NINE-I], Boris Böll [iCHOP, NINE-I], Matthias Kochanek [iCHOP, NINEI], Alexander Shimabukuro-Vornhagen [iCHOP, NINE-I]), University Hospital Essen (Tobias Liebregts [iCHOP, NINEI]), University Medical Center Hamburg-Eppendorf (Stefan Braune [iCHOP, NINE-I], Valentin Fuhrmann [iCHOP, NINE-I], Stefan Kluge [iCHOP, NINE-I]), Hannover Medical School (Gernot Beutel [iCHOP, NINE-I], Catherina Lueck [iCHOP, NINE-I]), University Hospital Muenster (Pia Lebiedz [iCHOP, NINE-I]), LMU Munich (Hans-Joachim Stemmler [iCHOP, NINE-I], Johanna Tischer [iCHOP, NINE-I]); Norway: Oslo University Hospital (Andreas Barrat-Due [NINEI], Yngvar Fløisand [NINE-I], Morten Svalebjørg [NINE-I]).

\section{REFERENCES}

1. Pasquini MC ZX: Current Uses and Outcomes of Hematopoietic Stem Cell Transplantation: CIBMTR Summary Slides. 2016. Available at: https://www.cibmtr.org/ReferenceCenter/SlidesReports/ SummarySlides/pages/index.aspx. Accessed May 17, 2016

2. Passweg JR, Baldomero $H$, Bader $P$, et al: Hematopoietic stem cell transplantation in Europe 2014: More than 40000 transplants annually. Bone Marrow Transplant 2016; 51:786-792

3. Yadav H, Nolan ME, Bohman JK, et al: Epidemiology of acute respiratory distress syndrome following hematopoietic stem cell transplantation. Crit Care Med 2016; 44:1082-1090

4. Ranieri VM, Rubenfeld GD, Thompson BT, et al: Acute respiratory distress syndrome: The Berlin definition. JAMA 2012; 307:2526-2533

5. Wohlfarth P, Ullrich R, Staudinger T, et al; Arbeitsgruppe für hämatoonkologische Intensivmedizin der Österreichischen Gesellschaft für Internistische und Allgemeine Intensivmedizin und Notfallmedizin (ÖGIAIN): Extracorporeal membrane oxygenation in adult patients with hematologic malignancies and severe acute respiratory failure. Crit Care 2014; 18:R20

6. Hsiao LT, Chung FP, Chiou TJ, et al: The limitations of extracorporeal membrane oxygenation as a bridge to allogeneic hematopoietic stem cell transplantation. Intensive Care Med 2014; 40:1971-1972

7. Liao WI, Tsai SH, Chiu SK: Successful use of extracorporeal membrane oxygenation in a hematopoietic stem cell transplant patient with idiopathic pneumonia syndrome. Respir Care 2013; 58:e6-e10

8. Koinuma T, Nunomiya S, Wada M, et al: Concurrent treatment with a tumor necrosis factor-alpha inhibitor and veno-venous extracorporeal membrane oxygenation in a post-hematopoietic stem cell transplant patient with idiopathic pneumonia syndrome: A case report. J Intensive Care $2014 ; 2: 48$

9. Kang HS, Rhee CK, Lee HY, et al: Clinical outcomes of extracorporeal membrane oxygenation support in patients with hematologic malignancies. Korean J Intern Med 2015; 30:478-488 
10. Gow KW, Wulkan ML, Heiss KF, et al: Extracorporeal membrane oxygenation for support of children after hematopoietic stem cell transplantation: The Extracorporeal Life Support Organization experience. J Pediatr Surg 2006; 41:662-667

11. Schmidt M, Bréchot N, Combes A: Ten situations in which ECMO is unlikely to be successful. Intensive Care Med 2016; 42:750-752

12. Staudacher DL, Bode C, Wengenmayer T: Four situations in which ECMO might have a chance. Intensive Care Med 2016; 42:1305-1306

13. Vincent JL, Moreno R, Takala J, et al: The SOFA (Sepsis-related Organ Failure Assessment) score to describe organ dysfunction/failure. On behalf of the Working Group on Sepsis-Related Problems of the European Society of Intensive Care Medicine. Intensive Care Med 1996; 22:707-710

14. Le Gall JR, Lemeshow S, Saulnier F: A new Simplified Acute Physiology Score (SAPS II) based on a European/North American multicenter study. JAMA 1993; 270:2957-2963

15. Schellongowski $\mathrm{P}$, Benesch M, Lang $\mathrm{T}$, et al: Comparison of three severity scores for critically ill cancer patients. Intensive Care Med 2004; 30:430-436

16. Charlson ME, Pompei $P$, Ales KL, et al: A new method of classifying prognostic comorbidity in longitudinal studies: Development and validation. J Chronic Dis 1987; 40:373-383

17. Murray JF, Matthay MA, Luce JM, et al: An expanded definition of the adult respiratory distress syndrome. Am Rev Respir Dis 1988; 138:720-723

18. Amato MB, Meade MO, Slutsky AS, et al: Driving pressure and survival in the acute respiratory distress syndrome. N Engl J Med 2015; 372:747-755

19. Paden ML, Conrad SA, Rycus PT, et al: Extracorporeal Life Support Organization Registry Report 2012. ASAIO journal 2013; 59:202-210

20. Noah MA, Peek GJ, Finney SJ, et al: Referral to an extracorporeal membrane oxygenation center and mortality among patients with severe 2009 influenza $\mathrm{A}(\mathrm{H} 1 \mathrm{~N} 1)$. JAMA 2011; 306:1659-1668

21. Lengliné $E$, Chevret $S$, Moreau AS, et al: Changes in intensive care for allogeneic hematopoietic stem cell transplant recipients. Bone Marrow Transplant 2015; 50:840-845

22. Platon L, Amigues L, Ceballos $P$, et al: A reappraisal of ICU and long-term outcome of allogeneic hematopoietic stem cell transplantation patients and reassessment of prognosis factors: Results of a 5-year cohort study (2009-2013). Bone Marrow Transplant 2015; 51:256-261

23. Azoulay E, Lemiale V, Mokart D, et al: Acute respiratory distress syndrome in patients with malignancies. Intensive Care Med 2014; 40:1106-1114

24. Pène F, Aubron C, Azoulay E, et al: Outcome of critically ill allogeneic hematopoietic stem-cell transplantation recipients: A reappraisal of indications for organ failure supports. J Clin Oncol 2006; 24:643-649

25. Afessa B, Azoulay E: Critical care of the hematopoietic stem cell transplant recipient. Crit Care Clin 2010; 26:133-150

26. Soubani AO, Kseibi E, Bander JJ, et al: Outcome and prognostic factors of hematopoietic stem cell transplantation recipients admitted to a medical ICU. Chest 2004; 126:1604-1611
27. Price KJ, Thall PF, Kish SK, et al: Prognostic indicators for blood and marrow transplant patients admitted to an intensive care unit. $A m \mathrm{~J}$ Respir Crit Care Med 1998; 158:876-884

28. Azoulay E, Soares M, Darmon M, et al: Intensive care of the cancer patient: Recent achievements and remaining challenges. Ann Intensive Care 2011; $1: 5$

29. Schmidt M, Bailey M, Sheldrake J, et al: Predicting survival after extracorporeal membrane oxygenation for severe acute respiratory failure. The Respiratory Extracorporeal Membrane Oxygenation Survival Prediction (RESP) score. Am J Respir Crit Care Med 2014; 189:1374-1382

30. Schmidt M, Zogheib E, Rozé H, et al: The PRESERVE mortality risk score and analysis of long-term outcomes after extracorporeal membrane oxygenation for severe acute respiratory distress syndrome. Intensive Care Med 2013; 39:1704-1713

31. Seggewiss $\mathrm{R}$, Einsele $\mathrm{H}$ : Immune reconstitution after allogeneic transplantation and expanding options for immunomodulation: An update. Blood 2010; 115:3861-3868

32. Chi AK, Soubani AO, White AC, et al: An update on pulmonary complications of hematopoietic stem cell transplantation. Chest 2013; 144:1913-1922

33. Soubani AO, Miller KB, Hassoun PM: Pulmonary complications of bone marrow transplantation. Chest 1996; 109:1066-1077

34. Azoulay E, Mokart D, Lambert J, et al: Diagnostic strategy for hematology and oncology patients with acute respiratory failure: Randomized controlled trial. Am J Respir Crit Care Med 2010; 182:1038-1046

35. Staudinger T, Bojic A, Hermann A, et al: Long-term ECMO without anticoagulation in patients with severe thrombocytopenia. Abstract (P62) presented at the 5th Euro-ELSO Annual Congress, Glasgow, Scotland, UK, June 1-4, 2016

36. Bellani G, Laffey JG, Pham T, et al; LUNG SAFE Investigators; ESICM Trials Group: Epidemiology, patterns of care, and mortality for patients with acute respiratory distress syndrome in intensive care units in 50 countries. JAMA 2016; 315:788-800

37. Peek GJ, Mugford M, Tiruvoipati R, et al; CESAR trial collaboration: Efficacy and economic assessment of conventional ventilatory support versus extracorporeal membrane oxygenation for severe adult respiratory failure (CESAR): A multicentre randomised controlled trial. Lancet 2009; 374:1351-1363

38. Guérin C, Reignier J, Richard JC, et al; PROSEVA Study Group: Prone positioning in severe acute respiratory distress syndrome. N Engl J Med 2013; 368:2159-2168

39. Papazian L, Forel JM, Gacouin A, et al; ACURASYS Study Investigators: Neuromuscular blockers in early acute respiratory distress syndrome. N Engl J Med 2010; 363:1107-1116

40. Ventilation with lower tidal volumes as compared with traditional tidal volumes for acute lung injury and the acute respiratory distress syndrome. The Acute Respiratory Distress Syndrome Network. N Engl J Med 2000; 342:1301-1308

41. Hoeper MM, Wiesner O, Hadem J, et al: Extracorporeal membrane oxygenation instead of invasive mechanical ventilation in patients with acute respiratory distress syndrome. Intensive Care Med 2013; 39:2056-2057 